\title{
Lapatinib/Trastuzumab Regimen
}

National Cancer Institute

\section{Source}

National Cancer Institute. Lapatinib/Trastuzumab Regimen. NCI Thesaurus. Code

C138040.

A chemotherapy regimen consisting of lapatinib and trastuzumab, used as a treatment for recurrent and metastatic HER-2/neu-positive breast cancer. 